\title{
Pair correlation and equidistribution on manifolds
}

\author{
Jens Marklof ${ }^{1}$
}

Received: 28 February 2019 / Accepted: 6 June 2019 / Published online: 17 June 2019

(c) The Author(s) 2019

\section{Abstract}

This study is motivated by a series of recent papers that show that, if a given deterministic sequence in the unit interval has a Poisson pair correlation function, then the sequence is uniformly distributed. Analogous results have been proved for point sequences on higher-dimensional tori. The purpose of this paper is to describe a simple statistical argument that explains this observation and furthermore permits a generalisation to bounded Euclidean domains as well as compact Riemannian manifolds.

Keywords Pair correlation · Equidistribution $\cdot$ Poisson process

Mathematics Subject Classification $11 \mathrm{~K} 06 \cdot 11 \mathrm{~K} 45$

\section{Introduction}

A sequence of real numbers $\xi_{1}, \xi_{2}, \xi_{3}, \ldots$ in the unit interval [0,1] is called uniformly distributed if, for any subinterval $[a, b] \subset[0,1]$, we have

$$
\lim _{N \rightarrow \infty} \frac{\#\left\{j \leq N \mid \xi_{j} \in[a, b]\right\}}{N}=b-a .
$$

That is, the proportion of elements that fall into a given subinterval is asymptotic to its length. A classic example is the Kronecker sequence $\xi_{j}=\langle j \alpha\rangle$ (where $\langle\cdot\rangle$ denotes the fractional part), which is uniformly distributed if and only if $\alpha$ is irrational. Once uniform distribution of a sequence is established, it is natural to investigate statistical properties on finer scales. One of the simplest such statistics is pair correlation. We say the sequence $\left(\xi_{j}\right)_{j \in \mathbb{N}}$ in $[0,1]$ has a Poisson pair correlation, if for any bounded interval $[a, b] \subset \mathbb{R}$ we have

\section{Communicated by Adrian Constantin.}

\footnotetext{
$凶 \quad$ Jens Marklof

j.marklof@bristol.ac.uk

1 School of Mathematics, University of Bristol, Bristol BS8 1TW, UK
} 


$$
\lim _{N \rightarrow \infty} \frac{\#\left\{\left(j_{1}, j_{2}\right) \in[1, N]^{2} \mid \xi_{j_{1}}-\xi_{j_{2}} \in\left[\frac{a}{N}, \frac{b}{N}\right], j_{1} \neq j_{2}\right\}}{N}=b-a
$$

The average gap between the first $N$ elements $\xi_{1}, \ldots, \xi_{N} \in[0,1]$ is $\frac{1}{N}$, and so, by rescaling the interval to $\left[\frac{a}{N}, \frac{b}{N}\right]$, we indeed measure correlations in units of the average gap size. The reference to Poisson stems from the fact that the right hand side of (1.2) corresponds to the pair correlation of a Poisson point process in $\mathbb{R}$ of intensity one. What is more, the convergence (1.2) holds almost surely, if $\left(\xi_{j}\right)_{j \in \mathbb{N}}$ is a sequence of independent, uniformly distributed random variables in [0, 1]. Even for simple deterministic sequences, however, the convergence of pair correlation measures remains a significant challenge. For instance (1.2) is known to hold for $\xi_{j}=\left\langle j^{k} \alpha\right\rangle$ ( $k \geq 2$ a fixed integer) for Lebesgue-almost every $\alpha$ [16], and a lower bound on the Hausdorff dimension of permissible $\alpha$ has recently been established [3]. But so far there is not a single explicit example of $\alpha$, such as $\alpha=\sqrt{2}$ or $\alpha=\pi$, for which (1.2) holds; not even in the quadratic case $k=2[9,13,14]$. There has been significant recent progress in characterising the Poisson pair correlation (1.2) for general sequences $\xi_{j}=\left\langle a_{j} \alpha\right\rangle$, for Lebesgue-almost every $\alpha$, in terms of the additive energy of the integer coefficients $a_{j}$; cf. [2,5] and references therein. Explicit examples for which Poisson pair correlation (1.2) can be established include the fractional part of squareroots, i.e., $\xi_{j}=\left\langle j^{1 / 2}\right\rangle[6]$, and directions of points in a shifted Euclidean lattice [7]. Note that (1.2) fails for the Kronecker sequence for any choice of $\alpha$ [11,12]. Another interesting case of a uniformly distributed sequence is $\xi_{j}=\left\langle p_{j} \alpha\right\rangle$, where $p_{j}$ denotes the $j$ th prime and $\alpha$ is irrational: also here (1.2) fails to hold, for almost every $\alpha$ [20]. This illustrates the perhaps unsurprising fact: uniform distribution does not imply Poisson pair correlation.

In two independent papers, Aistleitner et al. [1] and Grepstad and Larcher [8] reversed the question and asked whether Poisson pair correlation (1.2) of a given sequence implies uniform distribution. The answer is yes, even under weaker hypotheses than (1.2), for sequences in the unit interval $[1,8,18]$. The same has been established for point sequences on higher-dimensional tori $[10,19]$. In the present paper we develop a statistical argument that permits a generalisation of these findings to bounded domains in $\mathbb{R}^{d}$ (Sect. 2) as well as compact Riemannian manifolds (Sect. 3; the special case of flat tori is discussed in the "Appendix"). Instead of point sequences, we furthermore consider the more general setting of triangular arrays, i.e., sequences of finite point sets with increasing cardinality.

\section{Bounded domains}

Let $\Omega \subset \mathbb{R}^{d}$ be bounded with vol $\partial \Omega=0$, where vol denotes the Lebesgue measure in $\mathbb{R}^{d}$. (All subsets of $\mathbb{R}^{d}$ in this paper are assumed to be Borel sets.) Consider the triangular array $\xi=\left(\xi_{i j}\right)_{i j}$ with coefficients $\xi_{i j} \in \Omega$ and indices $i, j \in \mathbb{N}, j \leq N_{i}$, for some given $N_{i} \in \mathbb{N}$ such that $N_{i}<N_{i+1}$. 
Example 1 Let $\Omega=[0,1]$. Take a real sequence $\left(\xi_{j}\right)_{j \in \mathbb{N}}$ in $[0,1]$ (as in the introduction) and set $\xi_{i j}=\xi_{j}$ for $j \leq N_{i}=i \in \mathbb{N}$. Sequences may thus be realised as special cases of a triangular arrays.

Example 2 Let $\Omega=B_{1}^{d}$ be the open unit ball centered at the origin. Take a sequence $\left(a_{j}\right)_{j \in \mathbb{N}}$ in $\mathbb{R}^{d}$ such that $\left\|a_{j}\right\| \rightarrow \infty$, and set $\xi_{i j}=T_{i}^{-1} a_{j}$, with $N_{i}=\#\left\{j \mid\left\|a_{j}\right\|<T_{i}\right\}$ and $T_{1}<T_{2}<\ldots \rightarrow \infty$ increasing sufficiently fast so that $N_{j+1}>N_{j}$.

We associate with the $i$ th row of $\xi$ the Borel probability measure $v_{i}$ on $\Omega$, defined by

$$
\nu_{i} f=\frac{1}{N_{i}} \sum_{j=1}^{N_{i}} f\left(\xi_{i j}\right),
$$

where $f \in \mathrm{C}_{b}(\Omega)$ (bounded and continuous). In other words, $v_{i}$ represents $N_{i}$ normalised point masses at the points $\xi_{i 1}, \ldots, \xi_{i N_{i}}$.

Given a Borel probability measure $\sigma$ on $\Omega$, we say the triangular array $\xi$ is equidistributed in $(\Omega, \sigma)$ if $v_{i}$ converges weakly to $\sigma$; that is,

$$
\lim _{i \rightarrow \infty} v_{i} f=\sigma f \text { for every } f \in \mathrm{C}_{b}(\Omega) .
$$

In the case of Example 1, equidistribution in $([0,1)$, vol) corresponds to the classical notion of uniform distribution discussed in the introduction.

Let $A: \operatorname{cl} \Omega \rightarrow \mathrm{GL}(d, \mathbb{R})$ be a continuous map. This means in particular that $\Delta(x)=|\operatorname{det} A(x)|$ is bounded above and below by positive constants. Define the finite Borel measure $\sigma$ on $\Omega$ by

$$
\sigma(d x)=\Delta(x) d x
$$

By multiplying $A$ with a suitable scalar constant, we may assume without loss of generality that $\sigma(\Omega)=1$.

The role of $A$ in this paper is to set a local frame, at each point $x \in \Omega$, relative to which we measure correlations in the array $\xi$. This is particularly relevant in Sect. 3, where we extend the present discussion to manifolds. The simplest example of $A$ to keep in mind for now is the constant function $A(x)=\operatorname{vol}(\Omega)^{-1 / d} I_{d}\left(I_{d}\right.$ is the identity matrix), so that $\sigma(d x)=\operatorname{vol}(\Omega)^{-1} d x$ is the uniform probability measure on $\Omega$.

Given an increasing sequence $M=\left(M_{i}\right)_{i}$ in $\mathbb{R}_{>0}$, the pair correlation measure $\rho_{i}$ of $\xi$ is defined by

$$
\rho_{i} f=\frac{M_{i}}{N_{i}^{2}} \sum_{\substack{j_{1}, j_{2}=1 \\ j_{1} \neq j_{2}}}^{N_{i}} f\left(M_{i}^{1 / d} A\left(\xi_{i j_{1}}\right)\left(\xi_{i j_{1}}-\xi_{i j_{2}}\right)\right)
$$

where $f \in \mathrm{C}_{c}^{+}\left(\mathbb{R}^{d}\right)$ (non-negative, continuous with compact support). The sequence $M$ determines the scale on which we measure correlations, and $A\left(\xi_{i j_{1}}\right)$ provides a local 
rescaling of length units near each point $\xi_{i j_{1}}$, relative to the density of the measure $\sigma$. We call the pair $(A, M)$ a scaling.

If equidistribution (2.2) is known for some probability measure $\sigma$ with continuous density $\Delta$, then the most canonical choice for $A$ is $A(x)=\Delta^{1 / d}(x) I_{d}$ and $M_{i}=N_{i}$, so that (2.4) captures correlations in units of the average Euclidean distance between the $\xi_{i j}$ near $x$, which is proportional to $\left(N_{i} \Delta(x)\right)^{-1 / d}$. The point of the present discussion is, however, that we do not assume equidistribution of the array $\xi$, and hence there is no a priori preferred choice of $A$ or $\sigma$.

Note that $\rho_{i}$ is a locally finite Borel measure on $\mathbb{R}^{d}$. It is not a probability measure. We equip the space of locally finite Borel measures on $\mathbb{R}^{d}$ with the vague topology, and say $\xi$ has limiting pair correlation measure $\rho$ for the scaling $(A, M)$, if $\rho_{i}$ converges vaguely to $\rho$. That is, if

$$
\lim _{i \rightarrow \infty} \rho_{i} f=\rho f \quad \text { for every } f \in \mathrm{C}_{c}^{+}\left(\mathbb{R}^{d}\right) .
$$

We say $\rho_{i}$ has a Poisson limit for the scaling $(A, M)$ if (2.5) holds with $\rho=$ vol. (The constant multiplier in this relation seems arbitrary, but is in fact determined by our scaling of $A$ such that $\sigma(\Omega)=1$.) In this case (2.5) is equivalent to the statement

$$
\lim _{i \rightarrow \infty} \rho_{i} D=\operatorname{vol} D \text { for every bounded } D \subset \mathbb{R}^{d} \text { with } \operatorname{vol} \partial D=0 \text {, }
$$

where

$$
\rho_{i} D=\frac{M_{i}}{N_{i}^{2}} \#\left\{\left(j_{1}, j_{2}\right) \in \mathbb{Z}_{\neq}^{2} \cap\left[1, N_{i}\right]^{2} \mid \xi_{i j_{1}}-\xi_{i j_{2}} \in M_{i}^{-1 / d} A\left(\xi_{i j_{1}}\right)^{-1} D\right\},
$$

and $\mathbb{Z}_{\neq}^{2}=\mathbb{Z}^{2} \backslash\{(j, j) \mid j \in \mathbb{Z}\}$.

We furthermore say $\rho_{i}$ has a sub-Poisson limit if

$$
\limsup _{i \rightarrow \infty} \rho_{i} f \leq \operatorname{vol} f \text { for every } f \in \mathrm{C}_{c}^{+}\left(\mathbb{R}^{d}\right),
$$

which again is equivalent to the corresponding statement for bounded $D \subset \mathbb{R}^{d}$ with vol $\partial D=0$.

In many applications one considers only the pair correlation with respect to the distance between points. We consider here $\operatorname{dist}(x, y)=\|x-y\|$, with $\|\cdot\|$ the Euclidean norm in $\mathbb{R}^{d}$. The corresponding pair correlation is a locally finite Borel measure on $\mathbb{R}_{\geq 0}$ defined by

$$
\widetilde{\rho}_{i} h=\frac{M_{i}}{N_{i}^{2}} \sum_{\substack{j_{1}, j_{2}=1 \\ j_{1} \neq j_{2}}}^{N_{i}} h\left(M_{i}^{1 / d}\left\|A\left(\xi_{i j_{1}}\right)\left(\xi_{i j_{1}}-\xi_{i j_{2}}\right)\right\|\right)
$$

for $h \in \mathrm{C}_{c}^{+}\left(\mathbb{R}_{\geq 0}\right)$. In the spatial statistics literature variants of this are often referred to as Ripley's $K$-function; cf. [15, Sect. 8.3]. 
Define the Borel measure $\omega$ on $\mathbb{R}_{\geq 0}$ by

$$
\omega[0, r]=r^{d} \operatorname{vol} B_{1}^{d},
$$

where $B_{1}^{d}$ is the open unit ball. We say $\widetilde{\rho}_{i}$ has a Poisson limit if it converges vaguely to $\omega$. Note that if $h \in \mathrm{C}_{c}^{+}\left(\mathbb{R}_{\geq 0}\right)$ then $f \in \mathrm{C}_{c}^{+}\left(\mathbb{R}^{d}\right)$ for $f(x)=h(\|x\|)$. Therefore the vague convergence $\rho_{i} \rightarrow \rho$ implies the vague convergence $\widetilde{\rho}_{i} \rightarrow \widetilde{\rho}$ with $\widetilde{\rho}$ defined by the relation $\tilde{\rho} h=\rho f$ with $f(x)=h(\|x\|)$. Thus if $\rho_{i}$ has a Poisson limit in the vague topology, then so does $\widetilde{\rho}_{i}$. We say $\tilde{\rho}_{i}$ has a sub-Poisson limit if

$$
\limsup _{i \rightarrow \infty} \widetilde{\rho}_{i} h \leq \omega h \text { for every } h \in \mathrm{C}_{c}^{+}\left(\mathbb{R}_{\geq 0}\right)
$$

The latter statement is equivalent to

$$
\limsup _{i \rightarrow \infty} \widetilde{\rho}_{i}[0, r] \leq r^{d} \text { vol } B_{1}^{d} \text { for every } r>0 \text {. }
$$

Theorem 1 Fix $A$ and $\sigma$ as defined above, and let $\xi$ be a triangular array in $\Omega$. Then the following holds.

(i) Suppose there is a sequence $M$ with $M_{i} \rightarrow \infty$ and $M_{i} \leq N_{i}$, such that $\tilde{\rho}_{i}$ has a sub-Poisson limit for the scaling $(A, M)$. Then $\xi$ is equidistributed in $(\Omega, \sigma)$.

(ii) Suppose $\xi$ is equidistributed in $(\Omega, \sigma)$. Then there is a sequence $M$ with $M_{i} \rightarrow \infty$ and $M_{i} \leq N_{i}$, such that $\rho_{i}$ has a Poisson limit for the scaling $(A, M)$.

It is well known that equidistribution does not imply a Poisson pair correlation at the scale $M_{i}=N_{i}$. (An elementary example is the triangular array in $[0,1]$ given by $\xi_{i j}=\frac{j}{N_{i}}$.) Furthermore, a Poisson pair correlation at this scale does not imply that other fine-scale statistics, such as the nearest-neighbour distribution, are Poisson $[4,6,7]$.

The proof of part (i) is split into four lemmas. For $x \in \mathbb{R}^{d}$, define the counting measure $\hat{\mu}_{i}^{x}$ on $\mathbb{R}^{d}$ by

$$
\hat{\mu}_{i}^{x} f=\sum_{j=1}^{N_{i}} f\left(M_{i}^{1 / d} A\left(\xi_{i j}\right)\left(\xi_{i j}-x\right)\right)
$$

where $f \in \mathrm{C}_{c}^{+}\left(\mathbb{R}^{d}\right)$. Denote by $\chi_{D}$ the indicator function of a bounded subset $D \subset \mathbb{R}^{d}$. Then

$\hat{\mu}_{i}^{x} D=\sum_{j=1}^{N_{i}} \chi_{D}\left(M_{i}^{1 / d} A\left(\xi_{i j}\right)\left(\xi_{i j}-x\right)\right)=\#\left\{j \leq N_{i} \mid \xi_{i j} \in x+M_{i}^{-1 / d} A\left(\xi_{i j}\right)^{-1} D\right\}$.

For $\epsilon>0$, let $\Omega_{\epsilon}=\Omega+B_{\epsilon}^{d}$ be the $\epsilon$-neighbourhood of $\Omega$, where $B_{\epsilon}^{d}$ is the open ball of radius $\epsilon$ centered at the origin. The Tietze extension theorem allows us to extend 
$\Delta$ to a continuous function $\mathbb{R}^{d} \rightarrow \mathbb{R}_{>0}$. We also extend $\sigma$ to a locally finite measure outside $\Omega$ via relation $(2.3)$.

It is convenient to work with the following normalised variant of $\hat{\mu}_{i}^{x}$,

$$
\mu_{i}^{x}=\frac{M_{i}}{N_{i}} \hat{\mu}_{i}^{x}
$$

Lemma 1 Fix a triangular array $\xi$, a sequence $M$ with $M_{i} \rightarrow \infty$ and $M_{i} \leq N_{i}$, and a bounded set $D \subset \mathbb{R}^{d}$. Then, for $\epsilon>0$,

$$
\lim _{i \rightarrow \infty} \int_{\Omega_{\epsilon}} \mu_{i}^{x} D \sigma(d x)=\operatorname{vol} D
$$

Proof Since $\Delta$ is uniformly continuous, we have

$$
\begin{aligned}
\int_{\Omega_{\epsilon}} \mu_{i}^{x} D \sigma(d x) & =\frac{M_{i}}{N_{i}} \sum_{j=1}^{N_{i}} \int_{\Omega_{\epsilon}} \chi_{D}\left(M_{i}^{1 / d} A\left(\xi_{i j}\right)\left(\xi_{i j}-x\right)\right) \Delta(x) d x \\
& =\frac{M_{i}}{N_{i}} \sum_{j=1}^{N_{i}} \int_{\Omega_{\epsilon}} \chi_{D}\left(M_{i}^{1 / d} A\left(\xi_{i j}\right)\left(\xi_{i j}-x\right)\right)\left(\Delta\left(\xi_{i j}\right)+o(1)\right) d x \\
& =\frac{1}{N_{i}} \sum_{j=1}^{N_{i}} \int_{M_{i}^{1 / d} A\left(\xi_{i j}\right)\left(\xi_{i j}-\Omega_{\epsilon}\right)} \chi_{D}(x)(1+o(1)) d x
\end{aligned}
$$

For $M_{i}$ sufficiently large, we have

$$
D \subset M_{i}^{1 / d} A\left(\xi_{i j}\right) B_{\epsilon}^{d} \subset M_{i}^{1 / d} A\left(\xi_{i j}\right)\left(\xi_{i j}-\Omega_{\epsilon}\right),
$$

since $\xi_{i j}+B_{\epsilon}^{d} \subset \Omega_{\epsilon}$. This implies (2.16).

We denote by $\mathrm{C}_{c}\left(\Omega^{\circ}\right)$ the class of continuous functions $\Omega \rightarrow \mathbb{R}$ with compact support in the interior $\Omega^{\circ}$ of $\Omega$.

Lemma 2 Fix a triangular array $\xi$, a sequence $M$ with $M_{i} \rightarrow \infty$ and $M_{i} \leq N_{i}$, and a bounded set $D \subset \mathbb{R}^{d}$ with vol $D>0$. If for every Borel probability measure $\lambda$ on $\Omega$ with density in $\mathrm{C}_{c}\left(\Omega^{\circ}\right)$ we have

$$
\lim _{i \rightarrow \infty} \int_{\Omega} \mu_{i}^{x} D \lambda(d x)=\operatorname{vol} D
$$

then $\xi$ is equidistributed. 
Proof Let $f \in \mathrm{C}_{c}\left(\Omega^{\circ}\right)$ be the density of $\lambda$ with respect to $\sigma$. Then (2.19) states explicitly that

$$
\lim _{i \rightarrow \infty} \frac{M_{i}}{N_{i}} \sum_{j=1}^{N_{i}} \int_{\Omega} \chi_{D}\left(M_{i}^{1 / d} A\left(\xi_{i j}\right)\left(\xi_{i j}-x\right)\right) f(x) \sigma(d x)=\operatorname{vol} D \int_{\Omega} f(x) \sigma(d x),
$$

which by linearity in fact holds for any $f \in \mathrm{C}_{c}\left(\Omega^{\circ}\right)$, not necessarily probability densities. Since $f$ and $A$ are uniformly continuous and $D$ is bounded, we have uniformly for $y \in \Omega$,

$$
\begin{aligned}
& \int_{\Omega} \chi_{D}\left(M_{i}^{1 / d} A(y)(y-x)\right) f(x) \sigma(d x) \\
& \quad=f(y)(1+o(1)) \int_{\Omega} \chi_{D}\left(M_{i}^{1 / d} A(y)(y-x)\right) \sigma(d x),
\end{aligned}
$$

and

$$
\int_{\Omega} \chi_{D}\left(M_{i}^{1 / d} A(y)(y-x)\right) \sigma(d x)=\operatorname{vol} D+o(1)
$$

uniformly for all $y \in \operatorname{supp} f$. (This follows from the same reasoning as in the proof of Lemma 1 , since supp $f$ avoids an $\epsilon$-neighbourhood of $\partial \Omega$, for some $\epsilon>0$.) Therefore,

$$
\frac{M_{i}}{N_{i}} \sum_{j=1}^{N_{i}} \int_{\Omega} \chi_{D}\left(M_{i}^{1 / d} A\left(\xi_{i j}\right)\left(\xi_{i j}-x\right)\right) f(x) \sigma(d x)=\frac{\operatorname{vol} D}{N_{i}} \sum_{j=1}^{N_{i}} f\left(\xi_{i j}\right)+o(1)
$$

Thus (2.20) implies for $f \in \mathrm{C}_{c}\left(\Omega^{\circ}\right)$

$$
\lim _{i \rightarrow \infty} \frac{1}{N_{i}} \sum_{j=1}^{N_{i}} f\left(\xi_{i j}\right)=\int_{\Omega} f(x) \sigma(d x)
$$

This relation can be extended to $f \in \mathrm{C}_{b}(\Omega)$ by noting that (2.24) holds trivially for every constant test function: Any $f \in \mathrm{C}_{b}(\Omega)$ can be approximated from below by a function in $\mathrm{C}_{c}\left(\Omega^{\circ}\right)$, and from above by a function in $\mathrm{C}_{c}\left(\Omega^{\circ}\right)$ plus a constant. This proves that $\xi$ is equidistributed.

Lemma 3 Fix a triangular array $\xi$ and a bounded set $D \subset \mathbb{R}^{d}$. If there is a sequence $M$ with $M_{i} \rightarrow \infty$ and $M_{i} \leq N_{i}$ such that

$$
\lim _{i \rightarrow \infty} \int_{\Omega}\left(\mu_{i}^{x} D-\operatorname{vol} D\right)^{2} \sigma(d x)=0
$$


then

$$
\lim _{i \rightarrow \infty} \int_{\Omega} \mu_{i}^{x} D \lambda(d x)=\operatorname{vol} D
$$

for every Borel probability measure $\lambda$ with square-integrable density (with respect to $\sigma)$.

Proof Let $f$ be the density of $\lambda$. By the Cauchy-Schwarz inequality,

$$
\begin{aligned}
& \left|\int_{\Omega}\left(\mu_{i}^{x} D-\operatorname{vol} D\right) \lambda(d x)\right| \\
& \quad \leq\left(\int_{\Omega} f(x)^{2} \sigma(d x)\right)^{1 / 2}\left(\int_{\Omega}\left(\mu_{i}^{x} D-\operatorname{vol} D\right)^{2} \sigma(d x)\right)^{1 / 2} .
\end{aligned}
$$

This converges to zero as $i \rightarrow \infty$, which proves (2.26).

Lemma 4 Fix a triangular array $\xi$, a sequence $M$ with $M_{i} \rightarrow \infty$ and $M_{i} \leq N_{i}$, and a bounded subset $D \subset \mathbb{R}^{d}$ with vol $\partial D=0$. Set

$$
f(x)=\operatorname{vol}((D+x) \cap D) .
$$

Then $f \in \mathrm{C}_{c}^{+}\left(\mathbb{R}^{d}\right)$ and we have, for $\epsilon>0$,

$$
\int_{\Omega_{\epsilon}}\left(\mu_{i}^{x} D-\operatorname{vol} D\right)^{2} \sigma(d x)=\rho_{i} f-(\operatorname{vol} D)^{2}\left(2-\sigma\left(\Omega_{\epsilon}\right)\right)+\frac{M_{i}}{N_{i}} \operatorname{vol} D+o(1)
$$

Proof By Lemma 1,

$$
\int_{\Omega_{\epsilon}} \mu_{i}^{x} D \sigma(d x)=\operatorname{vol} D+o(1)
$$

and so

$$
\int_{\Omega_{\epsilon}}\left(\mu_{i}^{x} D-\operatorname{vol} D\right)^{2} \sigma(d x)=\int_{\Omega_{\epsilon}}\left(\mu_{i}^{x} D\right)^{2} \sigma(d x)-(\operatorname{vol} D)^{2}\left(2-\sigma\left(\Omega_{\epsilon}\right)\right)+o(1) .
$$


Furthermore, by the same reasoning as in the proof of Lemma 1,

$$
\begin{aligned}
& \int_{\Omega_{\epsilon}}\left(\mu_{i}^{x} D\right)^{2} \sigma(d x) \\
& =\frac{M_{i}^{2}}{N_{i}^{2}} \sum_{j_{1}, j_{2}=1}^{N_{i}} \int_{\Omega_{\epsilon}} \chi_{D}\left(M_{i}^{1 / d} A\left(\xi_{i j_{1}}\right)\left(\xi_{i j_{1}}-x\right)\right) \chi_{D}\left(M_{i}^{1 / d} A\left(\xi_{i j_{2}}\right)\left(\xi_{i j_{2}}-x\right)\right) \sigma(d x) \\
& =\frac{M_{i}}{N_{i}^{2}} \sum_{j_{1}, j_{2}=1}^{N_{i}} \int_{\mathbb{R}^{d}} \chi_{D}(x) \chi_{D}\left(x-M_{i}^{1 / d} A\left(\xi_{i j_{1}}\right)\left(\xi_{i j_{1}}-\xi_{i j_{2}}\right)\right) d x+o(1)
\end{aligned}
$$

The summation over distinct indices $j_{1} \neq j_{2}$ yields $\rho_{i} f$ with $f$ as defined in (2.28). The summation over $j_{1}=j_{2}$ yields $\frac{M_{i}}{N_{i}}$ vol $D$.

The function $f$ is compactly supported, since $D$ is bounded. To prove continuity, note that for $\|x-y\|<\epsilon,|f(x)-f(y)|$ is bounded above by the volume of the $\epsilon$-neighbourhood of $\partial D$. Continuity of $f$ is therefore implied by the assumption $\operatorname{vol} \partial D=0$.

Proof of Theorem 1 (i) Assume that $\widetilde{\rho}_{i}$ has a sub-Poisson limit for some sequence $M$ with $M_{i} \leq N_{i}$. It follows from (2.12) that, for any $\delta>0, \widetilde{\rho}_{i}$ also has a sub-Poisson limit for the scaling $\left(A, M^{\delta}\right)$ defined by $M_{i}^{\delta}=\delta M_{i}$. To highlight the $\delta$-dependence we write $\widetilde{\rho}_{i}=\widetilde{\rho}_{i}^{\delta}$.

Let $D=B_{1}^{d}$, and $f$ as defined in (2.28). $f(x)=\operatorname{vol}\left(\left(B_{1}^{d}+x\right) \cap B_{1}^{d}\right)$, and note that $f(x)=h(\|x\|)$ where $h(r)=\operatorname{vol}\left(\left(B_{1}^{d}+r e_{0}\right) \cap B_{1}^{d}\right)$ with $e_{0}$ an arbitrary choice of unit vector. The function $h$ is continuous and compactly supported on $\mathbb{R}_{\geq 0}$, with $h(0)=\operatorname{vol} B_{1}^{d}$. By assumption $\widetilde{\rho}_{i}^{\delta}$ has a sub-Poisson limit. Hence

$$
\limsup _{i \rightarrow \infty} \widetilde{\rho}_{i}^{\delta} h \leq \omega h
$$

and so

$$
\limsup _{i \rightarrow \infty} \rho_{i}^{\delta} f \leq \int_{\mathbb{R}^{d}} f(x) d x=(\operatorname{vol} D)^{2}
$$

With this, Lemma 4 shows that, for any $\epsilon, \delta>0$,

$$
\limsup _{i \rightarrow \infty} \int_{\Omega}\left(\mu_{i}^{x} D-\operatorname{vol} D\right)^{2} \sigma(d x) \leq\left(\sigma\left(\Omega_{\epsilon}\right)-1\right)(\operatorname{vol} D)^{2}+\delta \operatorname{vol} D
$$

Since vol $\partial \Omega=0$ and thus $\sigma(\partial \Omega)=0$, we have $\sigma\left(\Omega_{\epsilon}\right) \rightarrow \sigma(\Omega)=1$ as $\epsilon \rightarrow 0$. Thus there is a sequence of $\delta_{i} \rightarrow 0$, such that for the scaling $\left(A, M^{\prime}\right)$ given by $M_{i}{ }^{\prime}=\delta_{i} M_{i}$ we have

$$
\limsup _{i \rightarrow \infty} \int_{\Omega}\left(\mu_{i}^{x} D-\operatorname{vol} D\right)^{2} \sigma(d x)=0
$$


This confirms the hypothesis of Lemma 3 for the sequence $M^{\prime}$. Lemma 3 in turn establishes the assumption for Lemma 2, which completes the proof of claim (i).

Proof of Theorem 1 (ii) Since $\xi$ is equidistributed in $(\Omega, \sigma)$ we have, for $\psi \in \mathrm{C}_{b}(\Omega \times$ $\Omega)$,

$$
\lim _{i \rightarrow \infty} \frac{1}{N_{i}^{2}} \sum_{j_{1}, j_{2}=1}^{N_{i}} \psi\left(\xi_{i j_{1}}, \xi_{i j_{2}}\right)=\int_{\Omega \times \Omega} \psi\left(x_{1}, x_{2}\right) \sigma\left(d x_{1}\right) \sigma\left(d x_{2}\right)
$$

Since $\psi$ is bounded, the above statement remains valid with the diagonal terms $j_{1}=j_{2}$ removed. For fixed $M_{0}>0$ and $f \in \mathrm{C}_{c}^{+}\left(\mathbb{R}^{d}\right)$, apply this asymptotics with the choice $\psi\left(x_{1}, x_{2}\right)=M_{0} f\left(M_{0}^{1 / d} A\left(x_{1}\right)\left(x_{1}-x_{2}\right)\right)$, which is bounded continuous. This yields,

$$
\begin{aligned}
& \lim _{i \rightarrow \infty} \frac{M_{0}}{N_{i}^{2}} \sum_{\substack{j_{1}, j_{2}=1 \\
j_{1} \neq j_{2}}}^{N_{i}} f\left(M_{0}^{1 / d} A\left(\xi_{i j_{1}}\right)\left(\xi_{i j_{1}}-\xi_{i j_{2}}\right)\right) \\
& =M_{0} \int_{\Omega \times \Omega} f\left(M_{0}^{1 / d} A\left(x_{1}\right)\left(x_{1}-x_{2}\right)\right) \sigma\left(d x_{1}\right) \sigma\left(d x_{2}\right) .
\end{aligned}
$$

The right hand side can be written as

$$
\begin{aligned}
& M_{0} \int_{\Omega \times \Omega} f\left(M_{0}^{1 / d} A\left(x_{1}\right)\left(x_{1}-x_{2}\right)\right) \Delta\left(x_{1}\right) \Delta\left(x_{2}\right) d x_{1} d x_{2} \\
& =\int_{\Omega}\left(\int_{M_{0}^{1 / d}\left(\Omega-x_{2}\right)} f\left(A\left(M_{0}^{-1 / d} x_{1}+x_{2}\right) x_{1}\right) \Delta\left(M_{0}^{-1 / d} x_{1}+x_{2}\right) \Delta\left(x_{2}\right) d x_{1}\right) d x_{2} .
\end{aligned}
$$

Since $f, A$ are continuous and $\Omega$ has boundary of Lebesgue measure zero, this expression converges, as $M_{0} \rightarrow \infty$, to

$$
\int_{\Omega} \int_{\mathbb{R}^{d}} f\left(A\left(x_{2}\right) x_{1}\right) \Delta\left(x_{2}\right)^{2} d x_{1} d x_{2}=\int_{\Omega} \int_{\mathbb{R}^{d}} f\left(x_{1}\right) d x_{1} \sigma\left(d x_{2}\right)=\operatorname{vol} f .
$$

This proves that there is a slowly growing sequence $M_{i} \rightarrow \infty$ such that

$$
\lim _{i \rightarrow \infty} \frac{M_{i}}{N_{i}^{2}} \sum_{\substack{j_{1}, j_{2}=1 \\ j_{1} \neq j_{2}}}^{N_{i}} f\left(M_{i}^{1 / d} A\left(\xi_{i j_{1}}\right)\left(\xi_{i j_{1}}-\xi_{i j_{2}}\right)\right)=\operatorname{vol} f
$$

which proves part (ii) of the theorem. 


\section{Riemannian manifolds}

Let $(\mathcal{M}, g)$ be a compact Riemannian manifold with metric $g$. We denote by $\operatorname{vol}_{g}$ the corresponding Riemannian volume, and normalise $g$ such that $\operatorname{vol}_{g} \mathcal{M}=1$. The geodesic distance between $x, y \in \mathcal{M}$ is denoted dist $g(x, y)$. Now consider a triangular array $\xi$ with coefficients in $\mathcal{M}$, and define the corresponding pair correlation measure by

$$
\rho_{i}^{(g)} h=\frac{M_{i}}{N_{i}^{2}} \sum_{\substack{j_{1}, j_{2}=1 \\ j_{1} \neq j_{2}}}^{N_{i}} h\left(M_{i}^{1 / d} \operatorname{dist}_{g}\left(\xi_{i j_{1}}, \xi_{i j_{2}}\right)\right) .
$$

In other words, for $r>0$,

$$
\rho_{i}^{(g)}[0, r]=\frac{M_{i}}{N_{i}^{2}} \#\left\{\left(j_{1}, j_{2}\right) \in \mathbb{Z}_{\neq}^{2} \cap\left[1, N_{i}\right]^{2} \mid \operatorname{dist}_{g}\left(\xi_{i j_{1}}, \xi_{i j_{2}}\right) \leq M_{i}^{-1 / d} r\right\} .
$$

We say $\rho_{i}^{(g)}$ has a Poisson limit for the scaling $M$ if it converges vaguely to $\omega$, with $\omega$ as defined in (2.10) (with vol still the Lebesgue measure in $\mathbb{R}^{d}$ ), and similarly say it has a sub-Poisson limit if for every $h \in \mathrm{C}_{c}^{+}\left(\mathbb{R}_{\geq 0}\right)$

$$
\limsup _{i \rightarrow \infty} \rho_{i}^{(g)} h \leq \omega h
$$

which is equivalent to the statement

$$
\limsup _{i \rightarrow \infty} \rho_{i}^{(g)}[0, r] \leq r^{d} \text { vol } B_{1}^{d} \text { for every } r>0 .
$$

The following is a corollary of Theorem 1 .

Theorem 2 Let $(\mathcal{M}, g)$ be a compact Riemannian manifold, and $\xi$ a triangular array with coefficients in $\mathcal{M}$.

(i) Suppose there is a sequence $M$ with $M_{i} \rightarrow \infty$ and $M_{i} \leq N_{i}$, such that $\rho_{i}^{(g)}$ has a sub-Poisson limit for the scaling $M$. Then $\xi$ is equidistributed in $\left(\mathcal{M}, \mathrm{vol}_{g}\right)$.

(ii) Suppose $\xi$ is equidistributed in $\left(\mathcal{M}, \operatorname{vol}_{g}\right)$. Then there is a sequence $M$ with $M_{i} \rightarrow \infty$ and $M_{i} \leq N_{i}$, such that $\rho_{i}^{(g)}$ has a Poisson limit for the scaling $M$.

Part (i) is closely related to, but not implied by, the results in [17] for the choice $\rho_{i}^{(g)} h$ with $h(r)=\exp \left(-r^{2}\right)$.

Proof of (i) Consider an atlas $\left\{\left(U_{\alpha}, \varphi_{\alpha}\right) \mid \alpha \in \mathcal{A}\right\}$ with $\mathcal{A}$ finite. We take $\varphi_{\alpha}\left(U_{\alpha}\right) \subset \mathbb{R}^{d}$ to lie in the same copy of $\mathbb{R}^{d}$, arranged in such a way that the $\varphi_{\alpha}\left(U_{\alpha}\right)$ are pairwise disjoint. Now consider a partition of $\mathcal{M}$ by the bounded sets $V_{\beta}$ with $\beta \in \mathcal{B}$ and $\mathcal{B}$ finite, so that 


$$
\bigcup_{\beta \in \mathcal{B}} V_{\beta}=\mathcal{M}, \quad V_{\beta} \cap V_{\beta^{\prime}}=\emptyset \text { if } \beta \neq \beta^{\prime}, \quad \operatorname{vol}_{g} \partial V_{\beta}=0 .
$$

We assume the partition is sufficiently refined so that for $\beta \in \mathcal{B}$ there is a choice of $\alpha(\beta) \in \mathcal{A}$ such that $\mathrm{cl} V_{\beta} \subset U_{\alpha(\beta)}$. We set $\Omega_{\beta}=\varphi_{\alpha(\beta)} V_{\beta}$. The disjoint union

$$
\Omega=\bigcup_{\beta \in \mathcal{B}} \Omega_{\beta}
$$

is a bounded subset of $\mathbb{R}^{d}$ with vol $\partial \Omega=0$. Given a triangular array $\xi$ in $\mathcal{M}$ we define a corresponding array $\xi^{\prime}$, whose $i$ th row $\left(\xi_{i j}^{\prime}\right)_{j \leq N_{i}}$ is given by the elements in the set

$$
\bigcup_{\beta \in \mathcal{B}} \varphi_{\alpha(\beta)}\left(\left\{\xi_{i j} \mid j \leq N_{i}\right\} \cap V(\beta)\right) .
$$

By Gram-Schmidt orthonormalisation, there is a continuous function $A: \operatorname{cl} \Omega \rightarrow$ $\operatorname{GL}(d, \mathbb{R})$ such that the metric $g$ is given at $x \in U_{\alpha}$ by the positive definite bilinear form

$$
g_{x}(X, Y)=\left\langle A\left(\varphi_{\alpha} x\right) X, A\left(\varphi_{\alpha} x\right) Y\right\rangle,
$$

where $\langle\cdot, \cdot\rangle$ is the standard Euclidean inner product. With this choice, and the probability measure $\sigma$ defined as in (2.3), we see that the triangular array $\xi$ is equidistributed in $\left(\mathcal{M}, \operatorname{vol}_{g}\right)$ if and only if $\xi^{\prime}$ is equidistributed in $(\Omega, \sigma)$.

Let us now compare the pair correlation measure $\widetilde{\rho}_{i}$ for $\xi^{\prime}$ in $\Omega$ as defined in (2.9) with $\rho_{i}^{(g)}$. For $h \in \mathrm{C}_{c}^{+}\left(\mathbb{R}_{\geq 0}\right)$, we have

$$
h\left(M_{i}^{1 / d}\left\|A\left(\xi_{i j_{1}}\right)\left(\xi_{i j_{1}}^{\prime}-\xi_{i j_{2}}^{\prime}\right)\right\|\right)=0
$$

if $\xi_{i j_{1}}^{\prime} \in \Omega_{\beta}, \xi_{i j_{2}}^{\prime} \in \Omega_{\beta^{\prime}}$ with $\beta \neq \beta^{\prime}$ and $M_{i}$ is sufficiently large. This means that the pairs $\left(j_{1}, j_{2}\right)$ contributing to $\tilde{\rho}_{i}$ form a subset of those contributing to $\rho_{i}^{(g)}$. Furthermore, we have

$$
\operatorname{dist}_{g}(x, y) \sim\left\|A\left(\varphi_{\alpha} y\right)\left(\varphi_{\alpha} x-\varphi_{\alpha} y\right)\right\|
$$

for $\left\|\varphi_{\alpha} x-\varphi_{\alpha} y\right\| \rightarrow 0$. Both facts taken together imply, by the uniform continuity of $h \in \mathrm{C}_{c}^{+}\left(\mathbb{R}_{\geq 0}\right)$, that

$$
\limsup _{i \rightarrow \infty} \widetilde{\rho}_{i} h \leq \limsup _{i \rightarrow \infty} \rho_{i}^{(g)} h
$$

This shows that if $\rho_{i}^{(g)}$ has a sub-Poisson limit then so does $\widetilde{\rho}_{i}$. Theorem 1 tells us that therefore $\xi^{\prime}$ is equidistributed in $(\Omega, \sigma)$, and hence (as noted earlier) $\xi$ is equidistributed in $\left(\mathcal{M}, \operatorname{vol}_{g}\right)$. This yields claim (i). 
Proof of (ii) Since $\xi$ is equidistributed in $\left(\mathcal{M}, \operatorname{vol}_{g}\right)$ we have, for $\psi \in \mathrm{C}(\mathcal{M} \times \mathcal{M})$,

$$
\lim _{i \rightarrow \infty} \frac{1}{N_{i}^{2}} \sum_{j_{1}, j_{2}=1}^{N_{i}} \psi\left(\xi_{i j_{1}}, \xi_{i j_{2}}\right)=\int_{\mathcal{M} \times \mathcal{M}} \psi\left(x_{1}, x_{2}\right) \operatorname{vol}_{g}\left(d x_{1}\right) \operatorname{vol}_{g}\left(d x_{2}\right) .
$$

Since $\psi$ is bounded, the above statement remains valid with the diagonal terms $j_{1}=j_{2}$ removed. For fixed $M_{0}>0$ and $h \in \mathrm{C}_{c}^{+}\left(\mathbb{R}_{\geq 0}\right)$, apply this asymptotics with the choice $\psi\left(x_{1}, x_{2}\right)=M_{0} h\left(M_{0}^{1 / d} \operatorname{dist}_{g}\left(x_{1}, x_{2}\right)\right)$, which is bounded continuous. This yields,

$$
\begin{aligned}
& \lim _{i \rightarrow \infty} \frac{M_{0}}{N_{i}^{2}} \sum_{\substack{j_{1}, j_{2}=1 \\
j_{1} \neq j_{2}}}^{N_{i}} h\left(M_{0}^{1 / d} \operatorname{dist}_{g}\left(\xi_{i j_{1}}, \xi_{i j_{2}}\right)\right) \\
& =M_{0} \int_{\mathcal{M} \times \mathcal{M}} h\left(M_{0}^{1 / d} \operatorname{dist}\left(x_{1}, x_{2}\right)\right) \operatorname{vol}_{g}\left(d x_{1}\right) \operatorname{vol}_{g}\left(d x_{2}\right) .
\end{aligned}
$$

The limit $M_{0} \rightarrow 0$ can be calculated in local charts, which leads to the same calculation as in the proof of Theorem 1 (ii).

Open Access This article is distributed under the terms of the Creative Commons Attribution 4.0 International License (http://creativecommons.org/licenses/by/4.0/), which permits unrestricted use, distribution, and reproduction in any medium, provided you give appropriate credit to the original author(s) and the source, provide a link to the Creative Commons license, and indicate if changes were made.

\section{Appendix: Flat tori}

It is instructive to adapt the discussion in Sect. 2 to the case of a multidimensional torus $\mathbb{T}$. This provides an alternative approach to the results in [19]. We represent the torus as $\mathbb{T}=\mathbb{R}^{d} / \mathcal{L}$, with $\mathcal{L} \subset \mathbb{R}^{d}$ a Euclidean lattice of unit covolume (for example the integer lattice $\mathcal{L}=\mathbb{Z}^{d}$ ). The required modifications are as follows.

A. Replace $\Omega$ by $\mathbb{T}$ throughout Sect. 2, and note that $\mathrm{C}(\mathbb{T})=\mathrm{C}_{b}(\mathbb{T})=\mathrm{C}_{c}(\mathbb{T})$.

B. The coefficients of the triangular array are written as $\xi_{i j}+\mathcal{L} \in \mathbb{T}$ with $\xi_{i j} \in \mathbb{R}^{d}$.

C. Set for simplicity $A(x)=I_{d}$, so that $\sigma=$ vol is the uniform probability measure on $\mathbb{T}$. (It is of course possible to adapt the argument also for general continuous $A: \mathbb{T} \rightarrow \mathrm{GL}(d, \mathbb{R})$.)

D. The definition of the pair correlation measure $\rho_{i}$ in (2.4) is replaced by

$$
\rho_{i} f=\frac{M_{i}}{N_{i}^{2}} \sum_{\substack{j_{1}, j_{2}=1 \\ j_{1} \neq j_{2}}}^{N_{i}} \sum_{m \in \mathcal{L}} f\left(M_{i}^{1 / d}\left(\xi_{i j_{1}}-\xi_{i j_{2}}+m\right)\right)
$$


and (2.9) by

$$
\tilde{\rho}_{i} h=\frac{M_{i}}{N_{i}^{2}} \sum_{\substack{j_{1}, j_{2}=1 \\ j_{1} \neq j_{2}}}^{N_{i}} \sum_{m \in \mathcal{L}} h\left(M_{i}^{1 / d}\left\|\xi_{i j_{1}}-\xi_{i j_{2}}+m\right\|\right) .
$$

(Note that the pair correlation measure (3.1) for the Riemannian distance on $\mathbb{T}$,

$$
\operatorname{dist}_{g}(x, y)=\min _{m \in \mathcal{L}}\{\|x-y+m\|\},
$$

satisfies the relation $\widetilde{\rho}_{i} h=\rho_{i}^{(g)} h$, for $h \in \mathrm{C}_{c}^{+}\left(\mathbb{R}_{\geq 0}\right)$ and $M_{i}$ sufficiently large. $)$

E. The discussion around (2.16) is replaced by the following. For $x \in \mathbb{T}$, define the measure $\mu_{i}^{x}$ on $\mathbb{R}^{d}$ by

$$
\mu_{i}^{x} f=\frac{M_{i}}{N_{i}} \sum_{j=1}^{N_{i}} \sum_{m \in \mathcal{L}} f\left(M_{i}^{1 / d}\left(\xi_{i j}-x+m\right)\right)
$$

where $f \in \mathrm{C}_{c}^{+}\left(\mathbb{R}^{d}\right)$. That is, for a bounded subset $D \subset \mathbb{R}^{d}$, we have

$$
\begin{aligned}
\mu_{i}^{x} D & =\frac{M_{i}}{N_{i}} \sum_{j=1}^{N_{i}} \sum_{m \in \mathcal{L}} \chi_{D}\left(M_{i}^{1 / d}\left(\xi_{i j}-x+m\right)\right) \\
& =\frac{M_{i}}{N_{i}} \#\left\{j \leq N_{i} \mid \xi_{i j} \in x+M_{i}^{-1 / d} D+\mathcal{L}\right\}
\end{aligned}
$$

the second equality holds, if $M_{i}$ is sufficiently large so that $M_{i}^{-1 / d} D$ does not intersect any translate $M_{i}^{-1 / d} D+m$ with $m \in \mathcal{L} \backslash\{0\}$. Then

$$
\int_{\mathbb{T}} \mu_{i}^{x} D d x=\frac{M_{i}}{N_{i}} \sum_{j=1}^{N_{i}} \sum_{m \in \mathcal{L}} \int_{\mathbb{T}} \chi_{D}\left(M_{i}^{1 / d}\left(\xi_{i j}-x+m\right)\right) d x=\operatorname{vol} D
$$

F. In the statement of Lemma 4 no $\epsilon$ is needed, and (2.29) is replaced by the identity

$$
\int_{\mathbb{T}}\left(\mu_{i}^{x} D-\operatorname{vol} D\right)^{2} d x=\rho_{i} f-(\operatorname{vol} D)^{2}+\frac{M_{i}}{N_{i}} \operatorname{vol} D
$$


which follows from the following calculation, replacing (2.32),

$$
\begin{aligned}
\int_{\mathbb{T}}\left(\mu_{i}^{x} D\right)^{2} d x= & \frac{M_{i}^{2}}{N_{i}^{2}} \sum_{j_{1}, j_{2}=1}^{N_{i}} \sum_{m_{1}, m_{2} \in \mathcal{L}} \int_{\mathbb{T}} \chi_{D}\left(M _ { i } ^ { 1 / d } \left(\xi_{i j_{1}}\right.\right. \\
& \left.\left.-x+m_{1}\right)\right) \chi_{D}\left(M_{i}^{1 / d}\left(\xi_{i j_{2}}-x+m_{2}\right)\right) d x \\
= & \frac{M_{i}}{N_{i}^{2}} \sum_{j_{1}, j_{2}=1}^{N_{i}} \sum_{m \in \mathcal{L}} \int_{\mathbb{R}^{d}} \chi_{D}(x) \chi_{D}\left(x-M_{i}^{1 / d}\left(\xi_{i j_{1}}-\xi_{i j_{2}}+m\right)\right) d x
\end{aligned}
$$

G. For the proof of the second part of the theorem, we use instead

$$
\psi\left(x_{1}, x_{2}\right)=M_{0} \sum_{m \in \mathcal{L}} f\left(M_{0}^{1 / d}\left(x_{1}-x_{2}+m\right)\right)
$$

which is continuous on $\mathbb{T} \times \mathbb{T}$, with $f \in \mathrm{C}_{c}^{+}\left(\mathbb{R}^{d}\right)$ as before. The assumed equidistribution implies

$$
\begin{aligned}
& \lim _{i \rightarrow \infty} \frac{M_{0}}{N_{i}^{2}} \sum_{\substack{j_{1}, j_{2}=1 \\
j_{1} \neq j_{2}}}^{N_{i}} \sum_{m \in \mathcal{L}} f\left(M_{0}^{1 / d}\left(\xi_{i j_{1}}-\xi_{i j_{2}}+m\right)\right) \\
& =M_{0} \sum_{m \in \mathcal{L}} \int_{\mathbb{T} \times \mathbb{T}} f\left(M_{0}^{1 / d}\left(x_{1}-x_{2}+m\right)\right) d x_{1} d x_{2}
\end{aligned}
$$

which evaluates to vol $f$.

\section{References}

1. Aistleitner, C., Lachmann, T., Pausinger, F.: Pair correlations and equidistribution. J. Number Theory 182, 206-220 (2018)

2. Aistleitner, C., Lachmann, T., Technau, N.: There is no Khintchine threshold for metric pair correlations. arXiv:1802.02659

3. Aistleitner, C., Larcher, G., Lewko, M.: Additive energy and the Hausdorff dimension of the exceptional set in metric pair correlation problems. Israel J. Math. 222, 463-485 (2017)

4. Baddeley, A.J., Silverman, B.W.: A cautionary example on the use of second-order methods for analyzing point patterns. Biometrics 40, 1089-1093 (1984)

5. Bloom, T.F., Chow, S., Gafni, A., Walker, A.: Additive energy and the metric Poissonian property. Mathematika 64, 679-700 (2018)

6. El-Baz, D., Marklof, J., Vinogradov, I.: The two-point correlation function of the fractional parts of $\sqrt{n}$ is Poisson. Proc. AMS 143, 2815-2828 (2015)

7. El-Baz, D., Marklof, J., Vinogradov, I.: The distribution of directions in an affine lattice: two-point correlations and mixed moments. IMRN 1371-1400 (2015)

8. Grepstad, S., Larcher, G.: On pair correlation and discrepancy. Arch. Math. 109, 143-149 (2017)

9. Heath-Brown, D.R.: Pair correlation for fractional parts of $\alpha n^{2}$. Math. Proc. Camb. Philos. Soc. 148, 385-407 (2010) 
10. Hinrichs, A., Kaltenböck, L., Larcher, G., Stockinger, W., Ullrich, M.: On a multi-dimensional Poissonian pair correlation concept and uniform distribution. arXiv:1809.05672

11. Larcher, G., Stockinger, W.: Some negative results related to Poissonian pair correlation problems. arXiv:1803.052361

12. Marklof, J.: The n-point correlations between values of a linear form, with an appendix by Z. Rudnick. Ergod. Theory Dyn. Syst. 20, 1127-1172 (2000)

13. Marklof, J., Strömbergsson, A.: Equidistribution of Kronecker sequences along closed horocycles. Geom. Funct. Anal. 13, 1239-1280 (2003)

14. Marklof, J., Yesha, N.: Pair correlation for quadratic polynomials mod 1. Compos. Math. 154, 960-983 (2018)

15. Ripley, B.D.: Spatial Statistics. Wiley, Hoboken (1981)

16. Rudnick, Z., Sarnak, P.: The pair correlation function of fractional parts of polynomials. Commun. Math. Phys. 194, 61-70 (1998)

17. Steinerberger, S.: Localized quantitative criteria for equidistribution. Acta Arith. 180, 183-199 (2017)

18. Steinerberger, S.: Poissonian pair correlation and discrepancy. Indag. Math. 29, 1167-1178 (2018)

19. Steinerberger, S.: Poissonian pair correlation in higher dimensions. arXiv: 1812.10458

20. Walker, A.: The primes are not metric Poissonian. Mathematika 64, 230-236 (2018)

Publisher's Note Springer Nature remains neutral with regard to jurisdictional claims in published maps and institutional affiliations. 